\title{
Assessment of an infant whole-body plethysmograph using an infant lung function model
}

\author{
B. Reinmann*, J. Stocks*, U. Frey*
}

\begin{abstract}
Assessment of an infant whole-body plethysmograph using an infant lung function model. B. Reinmann, J. Stocks, U. Frey. C) ERS Journals Ltd 2001.

ABSTRACT: In order to facilitate international multicentre studies and improve the quality control of infant pulmonary function measurements, the European Respiratory Society-American Thoracic Society Task Force for infant lung function testing has recently developed specifications for standardized infant lung function equipment and software. A mechanical infant lung model analogue has been developed to assess whether infant lung function equipment is able to meet these requirements. However, the practical testing of infant lung function equipment using such models is highly complex because of the need to use very small pressure and flow changes, and the numerous potentially confounding factors associated with both the design of the device and the testing procedure.

The aim of this study was to determine whether the infant lung model is capable of assessing the overall function of an whole-body infant- plethysmograph, using the only infant plethysmograph that was commercially available at the time as an example.

The mechanical characteristics of the model such as vibrations or noise did not disturb the delicate plethysmographic measurements and thereby allowed a reliable assessment of the system. A series of tests revealed that the plethysmograph was able to measure airway resistance $1-3.5 \mathrm{kPa} \cdot \mathrm{L}^{-1} \cdot \mathrm{s}$ with an accuracy of $\pm 2.5 \%$ and lung volumes $75-300$ $\mathrm{mL}$ with an accuracy of $\pm 2.5 \%$ under in vitro conditions.

To conclude, the infant lung model is a useful means of assessing the overall in vitro performance of infant whole-body plethysmographs, but thermal, mechanical and frequency response characteristics of such a device must be taken into account when interpreting the results of such assessments.

Eur Respir J 2001; 17: 765-772.
\end{abstract}

*University Hospital of Berne, Inselspital, Switzerland. " Portex Anaesthesia, Intensive Care and Respiratory Medicine Unit, Institute of Child Health, London, UK.

Correspondence: U. Frey

Dept of Paediatrics

University Hospital of Berne

Inselspital

3010 Bern

Switzerland

Fax: 41316329484

\section{Keywords: Infant}

respiratory function test

respiratory mechanics

plethysmography

whole body

standardization

Received: September 81999

Accepted after revision December 29 2000
To facilitate international multicentre studies and improve the quality control of infant pulmonary function measurements, the European Respiratory Society (ERS)-American Thoracic Society (ATS) working group for infant lung function testing has recently developed specifications for standardized infant lung function equipment and software [1-7]. The assessment of infant lung mechanics requires higher technical demands of the equipment than when studying adults, because of smaller signal to noise ratio and higher respiratory rate. Whole-body infant-plethysmography (IWBP) $[4,8,9]$ is a particularly challenging technique with respect to these signal to noise and calibration problems, but remains an important method in that it allows simultaneous assessments of airway resistance (Raw) and functional residual capacity (FRCpleth) in these young subjects. The criteria which infant-plethysmographs should meet have been described recently $[4,8]$, as have general aspects of equipment and processing [1, 2]. Based on these guidelines, it should be possible to assess the mechanical and thermal properties of the plethysmograph itself, as well as the linearity and frequency response of the signal transducers.
While the testing of isolated components of plethysmographs has been described in detail in the literature [10-15], there has been no guidance as to how the overall performance of infant-plethysmographs should be assessed. The latter is very important since even if the physical properties of each component of the plethysmograph adhere to specified standards, their integrated function might fail or display unexpected problems.

To test the integrated overall performance of infant lung function devices, and particularly that of plethysmographs, a "mechanical model baby" which is partially able to simulate the breathing behaviour of infants of $2-12 \mathrm{~kg}$ body weight has recently been built [16]. However, the practical testing of infant lung function equipment using such a model is complex and reveals unexpected pitfalls. This is mainly due to the fact that the plethysmographic measurements in infants are potentially and critically influenced by factors such as signal to noise problems and thermal effects due to motor vibrations and heating effects of the pump when a model is placed within the plethysmograph.

The aim of this study was to investigate whether the infant lung model can be used to test the integrated performance of an infant plethysmograph by testing the 
only currently available commercial model (MasterScreen BabyBody; Erich Jaeger GmbH, Hoechberg, Germany). The authors particularly wanted to investigate whether an infant-plethysmograph can measure lung volumes, flow resistances and amplitude and phase relationships between two corresponding signals (pressure or flow, respectively) accurately, and to develop recommendations regarding optimal testing procedure when performing such in vitro assessments.

\section{Methods and results}

\section{Study design}

For clarity, the protocol is presented in four stages. For each section, the rationale, methods, results and a short summary are presented. Firstly, the influence of the model itself (e.g. vibrations, warming) on plethysmographic box pressure signals (signal to noise ratio) was assessed. Secondly, the accuracy with which an infant plethysmograph could measure a range of model lung volumes [16] at various breathing frequencies was determined. Thirdly, the accuracy with which an infant plethysmograph could measure a range of model airflow resistors [16] was assessed. Finally, the amplitude and phase relation of box volume ( $V$ box) versus pressure at the airway opening $(P \mathrm{ao})$ and $V$ box versus flow $\left(V^{\prime}\right)$ was tested, at $0.25-10 \mathrm{~Hz}[2,17]$.

\section{Effect of the infant lung model on the plethysmo- graphic signals}

Rationale. Box pressure measurements in infant-plethysmographs are prone to environmental noise that will influence the signal to noise ratio and the quality of the data. Any vibration or heat generated from the motor of an infant lung model will crucially influence measurements. The potential influence of the infant lung model on plethysmographic measurements were therefore assessed.

Methods. The infant model consisted of a computer driven linear motor with two different exchangeable pistons (Scireq Inc., Montreal, CA, USA) [18] and various volume and resistor elements. Its characteristics and accuracy have been described in the accompanying paper [16]. The composition of the infant lung model can vary depending on whether infant lung volumes, flows or airway resistances are to be simulated. The effects of the model vibrations on the box pressure signal were measured using a Honeywell pressure transducer (model $163 \mathrm{PC}$, range $\pm 1.25 \mathrm{kPa}$; Honeywell, Freeport, IL, USA) and a 12 bit ADboard. The heating effect of the model pump, when inside a closed plethysmograph, was measured over 20 min using an electronic thermometer (Impac Tastotherm-Hum RP2, Q+S Control AG, Oetwil, Switzerland).

Results. Vibrations of the lung model due to pump motor action did not cause any detectable box pressure changes. There was no heat production over
$20 \mathrm{~min}$ and hence no shift in baseline volume in a plethysmographic box.

In summary. Based on recent standards [1, 2, 4], the minimal expected volume displacement in the box during Raw measurements is $1 \mathrm{~mL}$ corresponding to a pressure change of $0.001 \mathrm{kPa}(\Delta P$ box $)$ in a $100 \mathrm{~L}$ chamber. The Sqirec linear motor pump did not cause significant signal to noise problems or baseline drift in this order of magnitude and was, therefore, suitable for use in an infant plethysmograph.

\section{Accuracy of in vitro volume measurements}

Rationale. FRC is an important physiological parameter in infants. During plethysmography, FRC is measured from the changes in box volume ( $\Delta V$ box $)$ and pressure at the airway opening $(P a)$ during an airway occlusion. Measuring small lung volumes in infant plethysmographs is difficult, since the ratio of the measured lung volume to plethysmographic box volume, which determines the amplitude of $\Delta V$ box and thus the signal to noise ratio, is relatively small. Although an infant lung model can potentially be used to determine the smallest measurable volume, such measurements are complex. For example, whereas gas compression during the FRC manoeuvre is isothermal in an infant, thermal effects during ventilation of the model volume elements, together with any pressure losses due to mechanical leaks in the model, must be considered [16]. Nevertheless, as described previously, the infant lung model can simulate effective lung model volumes $(\mathrm{Vm})$ of $\sim 50,75,100,150$ and $300 \mathrm{~mL}$ and thermal and leak effects can be taken into account [16].

A second problem that occurs during FRCpleth measurements concerns the time constant of the plethysmograph. Plethysmographs have an in-built, well-defined mechanical leak, which acts like a mechanical high pass filter. This leak allows equilibration of slow pressure changes due to warming of air that occurs when an infant is enclosed inside the plethysmograph. However, if the time constant of the box is very short, not only slow pressure changes, but also those due to breathing movements, will be at least partly attenuated, with subsequent underestimation of FRC. Thus, an ideal leak time constant has to be established that allows equilibration of temperature changes, while being long enough not to affect the lung volume measurements. An infant lung model can be used to determine this ideal time constant.

Methods. The lung model pump was placed inside the plethysmograph and was connected to the mask connection port of the pneumotachograph (PNT) (fig. 1a), using a connector that was specially designed to fit the Jaeger infant PNT. Pressure changes in the model were measured using the $P$ ao transducer supplied with the plethysmograph. Since measurements were being made on a lung model under ambient conditions, the automatic body temperature and pressure, saturated (BTPS) corrections of the plethysmograph were overridden before starting these measurements. 
a)

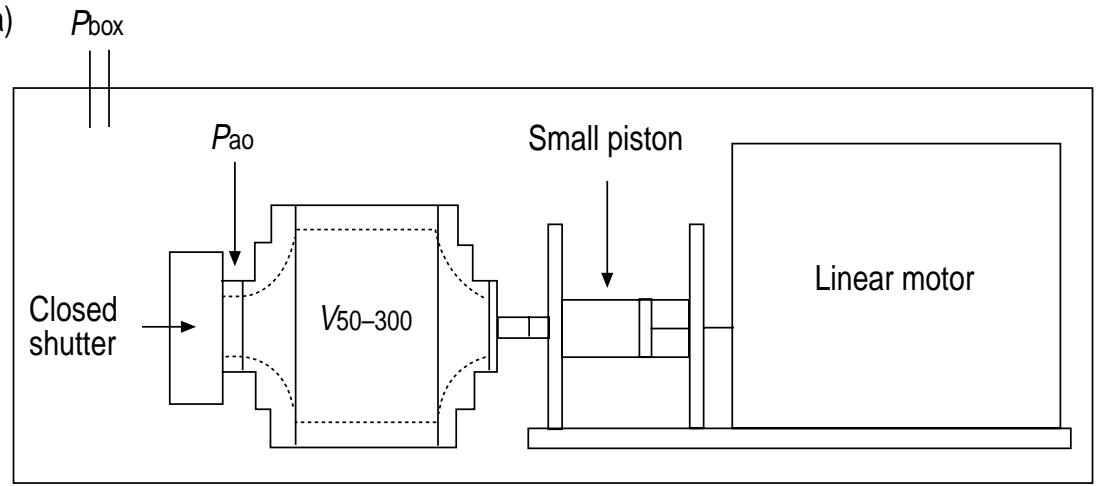

b)

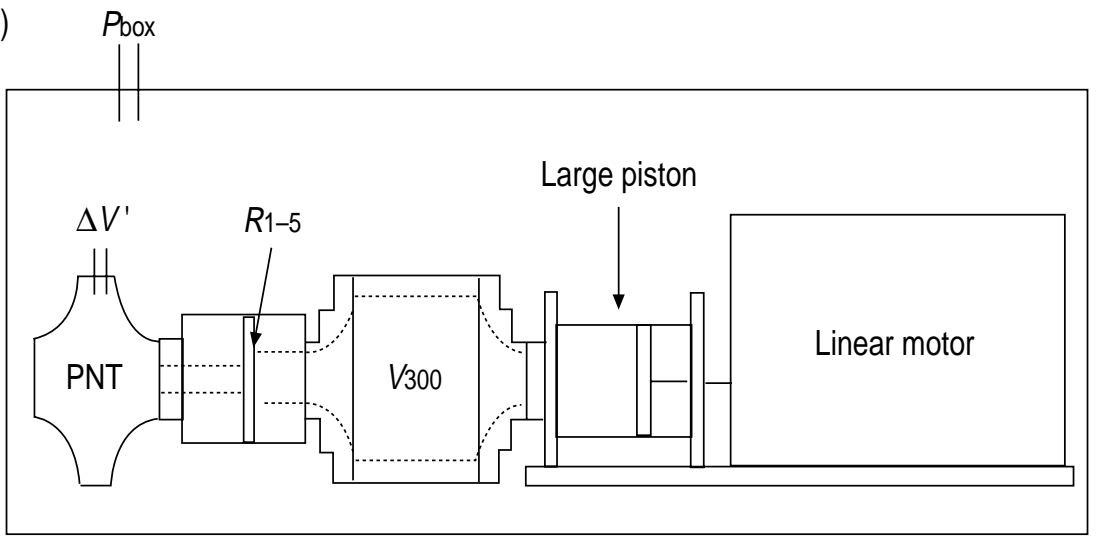

Fig. 1. - a) Set-up used to assess the accuracy with which an infant plethysmograph can measure lung volume. The lung model contains a series of different volume elements $\left(50-300 \mathrm{~mL} ; V_{50-300)}\right.$ which were connected to the mask connector and effective volume of the model was calculated from changes at the model opening $(P$ ao $)$ and box volume $(V$ box $)$. b) The set-up used to measure airway resistance in a plethysmograph using the infant lung model. The lung model consists of a given volume $\left(300 \mathrm{~mL} ; V_{300}\right)$ and resistors $R(1-$ $5.6 \mathrm{kPa} \cdot \mathrm{L}^{-1} \cdot \mathrm{s}$ ) connected to the pneumotachograph (PNT). $\Delta V^{\prime}$ : change in flow; Pbox: box pressure.

Specialized software (J-Scope; Erich Jaeger GmbH) was used to save the calibrated real-time signals during data recording. $V \mathrm{~m}$ was calculated from changes in $P$ ao and $V$ box as follows:

$$
V_{\mathrm{m}}=\left[P_{\mathrm{bar}} \times \Delta V_{\mathrm{box}} / \Delta P_{\mathrm{ao}}\right]-V_{\mathrm{ds}, \mathrm{app}}
$$

where $P$ bar is the barometric or ambient pressure, $\Delta P$ ao the change in airway opening pressure in the model and $V$ ds,app the apparatus dead space. These measurements were done in Würzburg, Germany at a barometric pressure of $98.0 \mathrm{kPa}$, a humidity of $50 \%$ and a temperature of $20^{\circ} \mathrm{C}$.

To assess whether the plethysmograph was capable of measuring infant lung volumes, 5 measurements were performed at each model volume (V50-300: 50, 75, 100,150 , and $300 \mathrm{~mL}$ ) [16] at a constant pumping frequency of $40 \mathrm{~min}^{-1}$ against the occlusion and a stroke volume of $0.5,1,1.5,2$ and $2.5 \mathrm{~mL}$, respectively. The results were compared to the known effective model volume in a modified Bland-Altman plot [19]. Secondly, in order to find the ideal leak time constant of the infant-plethysmograph, measurements of the model volume $V_{100}$ [16] were performed at pumping frequencies of $10,20,40,60,80,100$ and $120 \mathrm{~min}^{-1}$ using a stroke volume of $2 \mathrm{~mL}$ against the occlusion. At each frequency, the set of five measurements was then averaged (mean and 95\% confidence interval (CI)) and presented as \%pred of the model volume. These measurements were repeated at different half-life constants of the infant-plethysmograph $(4.2,9.9$ and $13.1 \mathrm{~s})$. Since the half-life constant represents the time taken for a given pressure to decay to $50 \%$, these correspond to time constants of $6.1,14.3$ and 18.9, respectively.

Results. Figure 2 shows mean and 95\% CI of the difference between expected and measured volumes plotted against the mean volume in a Bland-Altman plot. The two dashed lines represent the $\pm 2.5 \%$ accuracy range specified by the ATS-ERS standards [2]. Volumes $75-300 \mathrm{~mL}$ could be measured with an accuracy of $\pm 2.5 \%$ by the Jaeger plethysmograph. However, V50 was overestimated by $\sim 9 \%$, due to the very small pressure changes within the box of $<0.001$ $\mathrm{kPa}$ during the assessment of this element. The accuracy (mean, 95\% CI) with which the infant-plethysmograph could measure effective $V 100$ at different breathing frequencies in the presence of various halflife constants is illustrated in figure 3. The dashed lines represent the $\pm 2.5 \%$ error range specified in the standards [2]. It can be seen that lung volumes were measured accurately when a half-life of $9.9 \mathrm{~s}$ and 13.1 $\mathrm{s}$ were used. 


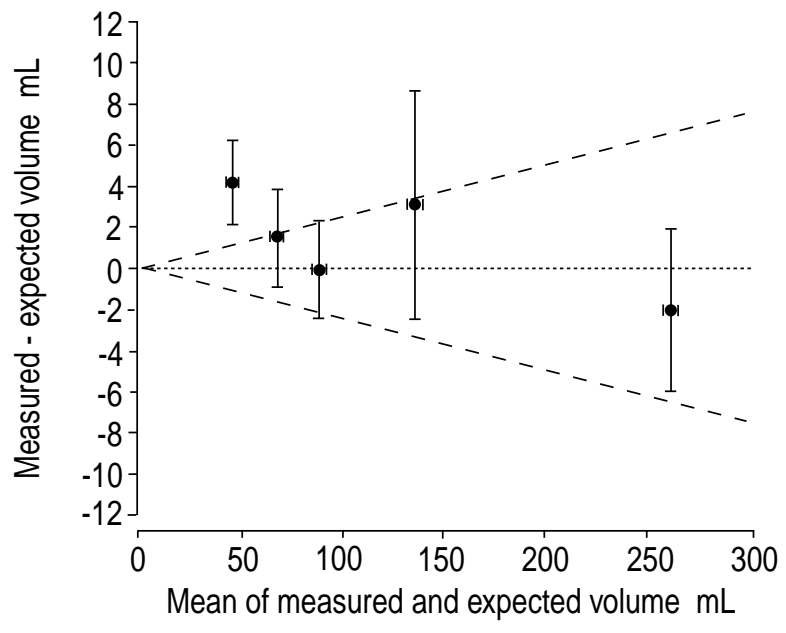

Fig. 2. - Accuracy of in vitro assessment of lung volume. These data were obtained at a frequency of $40 \mathrm{~min}^{-1}$ against the occlusion. The difference and $95 \%$ confidence interval between the expected and the measured volumes is plotted against the mean of these two values according to the method of Bland and Altman [19]. It can be seen that whereas the smallest volume (V50) was overestimated by $\sim 9 \%$, V75-300 were measured within $\pm 2.5 \%$, as represented by the two dashed lines.

In summary. Using the infant lung model with volume elements between 50 and $300 \mathrm{~mL}$, it was possible to test the accuracy of FRC measurements of infantplethysmographs in vitro according to the ERS-ATS standards $[1,2]$. A series of tests revealed that the Jaeger plethysmograph was able to measure model volumes $75-300 \mathrm{~mL}$ with an accuracy of $\pm 2.5 \%$. The plethysmograph was able to measure these effective $V \mathrm{~m}$ within an accuracy of $\pm 2.5 \%$ at all frequencies, but only if the half-life of the box was set to $\geqslant 10 \mathrm{~s}$ (equivalent to a time constant of 12.6 seconds). It should be noted that even if the model behaves polytropically, the expected pressure changes within the model and therefore the effective lung model volume can be measured accurately at frequencies 10-120 $\min ^{-1}$ using this approach. Volume measurements using the infant lung model thus helped to identify the ideal time constant of the infant plethysmograph.

\section{Assessing the resistance of the lung model}

Rationale. Airway resistance (Raw) is an important physiological parameter to describe airway obstruction. In an infant-plethysmograph, Raw is measured by relating changes in the box signal to the resulting flow at the airway opening. As previously described [16], the infant lung model is capable of reproducing pressure-flow curves from various screen resistors with a precision of $< \pm 2.5 \%$. However, measurements of model resistors within an infant plethysmograph are complex and require a carefully designed protocol.

Methods. To examine whether an infant plethysmograph is capable of measuring the resistance of such screens accurately, the model was placed within the plethysmograph and the model outlet was con- a)

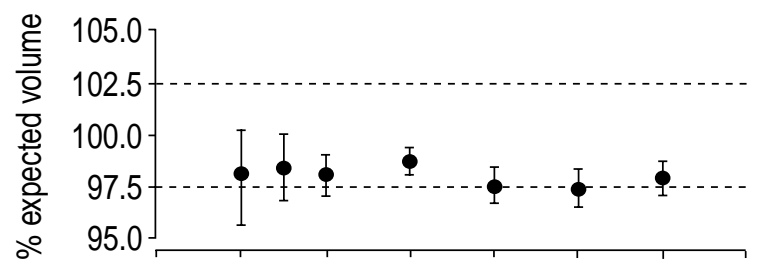

b)

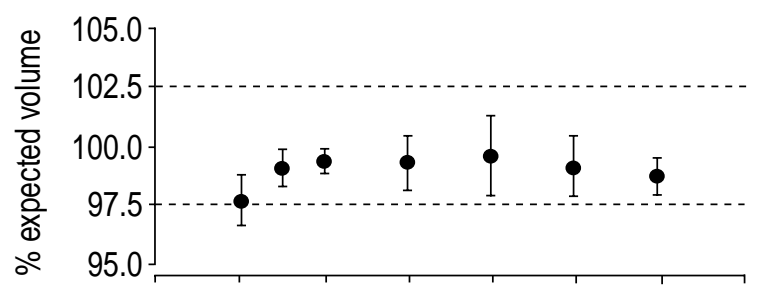

c)

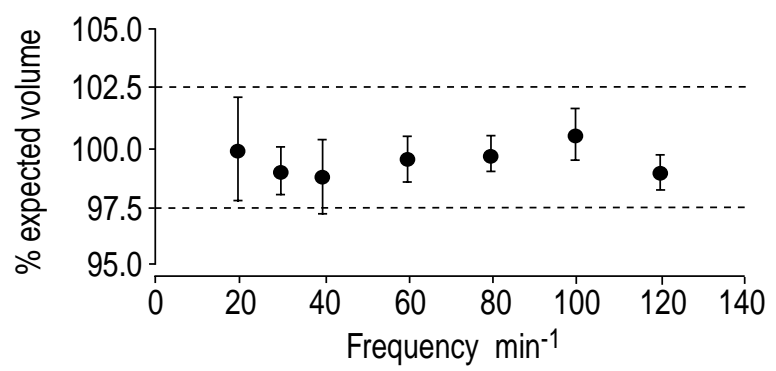

Fig. 3. - Accuracy of the infant plethysmograph in the presence of various half-life constants (a) 4.25; b) 9.95; and c) $13.1 \mathrm{~s}$ ) for the box (mean, 95\% confidence interval). V100 was measured at frequencies of $10-120 \mathrm{~min}^{-1}$ at different half-life constants. The dashed lines represent the $\pm 2.5 \%$ error range as specified by the American Thoracic Society - European Respiratory Society standards.

nected to the PNT (fig. 1). A $300 \mathrm{~mL}$ volume element (V300) was placed between the pump and the various resistors (Range: $1-3.5 \mathrm{kPa} \cdot \mathrm{L}^{-1} \cdot \mathrm{s}$ ). The insertion of this volume element, which acted as a capacitance, was necessary to achieve changes in box signal during ventilation of the model within the plethysmograph, that were of a similar magnitude to those observed in infants. $V 300$ had previously been shown not to introduce any significant phase lag between pressure and flow changes in the lung model below pumping frequencies of $120 \mathrm{~min}^{-1}$ [16].

The box was calibrated with the model in place, having previously overridden the automated Jaeger correction factors for body weight by setting the infant weight to its smallest value $(1 \mathrm{~kg})$. When calculating resistance, changes in box signal have to be calibrated in terms of alveolar pressure change by establishing the relationship between these two signals during an airway occlusion [8]. A similar procedure was performed for the lung model, the stroke volume during the occlusion being adjusted so that $\Delta P$ ao during the occlusion remained well within the range of the pressure transducer. The model was then opened and set to cycle at $1 \mathrm{~Hz}$ with a tidal volume of $80 \mathrm{~mL}$. A relatively large tidal volume was necessary during 
resistance measurements to ensure adequate changes in flow and $V$ box when using the lower resistance screens. The Jaeger software calculates the mean of 5 specific effective resistance measurements $(\mathrm{s} R$ eff $=R$ eff $\times V 300)$ of the screen resistor elements (examples used: $R 1,2,4,5$ ) from the phase angle between $V$ box and flow, wherein $\mathrm{s} R$ eff represents the specific resistance measured over the entire "breath", using all the available Vbox/flow data points. sReff was corrected for an apparatus resistance of $0.38 \mathrm{kPa} \cdot \mathrm{L}^{-1} \cdot \mathrm{s}^{-1}$. The resistance of each screen was calculated as $R$ eff $=\mathrm{s} R$ eff $/ V 300$.

The physical characteristics of the resistor elements $(R 1,2,4,5)$ have been described previously [16]. The measured values were compared with expected values based on the known resistances at specified flows and the effective volume of the model at the time of measurement.

Results. The accuracy with which the infant plethysmograph measured one particular screen resistor $(R 1)$ is presented in figure 4 which shows five single $V$ box versus flow loops, together with the calculated mean values of $\mathrm{s} R$ eff and Reff. The mean resistance for each of the four resistances, together with percentage errors relative to expected values, are presented in table 1. All resistances were calculated over the flow range of $\pm 0.1 \mathrm{~L} \cdot \mathrm{s}^{-1}$. $R 1$ showed the biggest error probably due to a very small box signal during the resistance measurement.

In summary. By using the infant lung model with various exchangeable screen resistors, it was possible to test the in vitro accuracy of resistance measurements of an infant-plethysmograph according to ERS-ATS standards [1, 2, 4]. There was no drift or noise introduced by the model, provided a volume

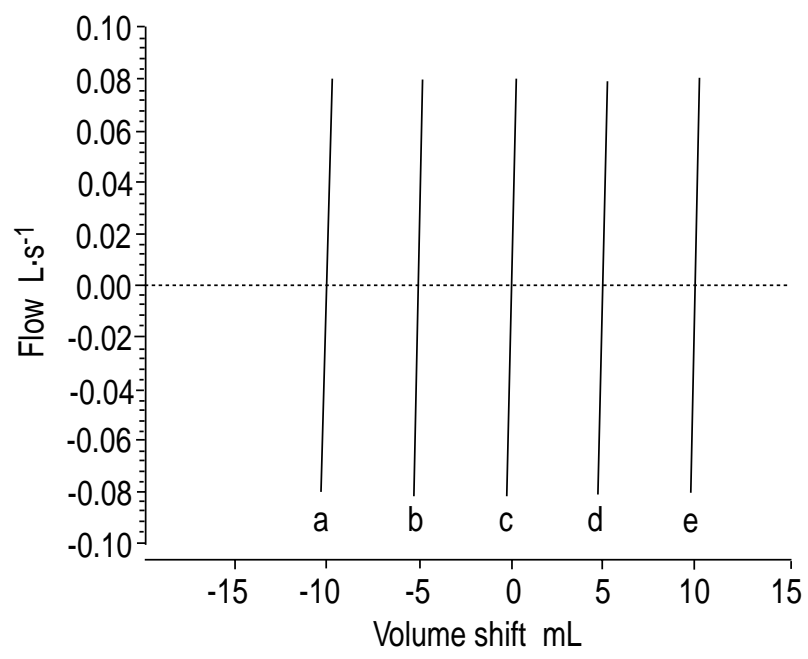

Fig. 4. - A representative example $\left(R_{1}\right)$ of the performance of the infant plethysmograph while measuring the infant lung model, showing changes in the box volume plotted against the corresponding flow changes. It can be seen that there is only discrete looping at a breathing frequency of $1 \mathrm{~Hz}\left(60 \mathrm{~min}^{-1}\right)$ and airway resistance can easily be determined from these measurements. Specific effective resistance and effective resistance, respectively, are as follows: a) $0.35,1.30$; b) $0.30,1.10$; c) $0.32,1.18$; d) $0.31,1.15$; e) $0.31,1.14$
Table 1. - Expected resistance, measured resistance and percentage error of $R 1,2,4,5$

\begin{tabular}{lccc}
\hline & $\begin{array}{c}\text { Expected } \\
\text { resistance } \\
\mathrm{kPa} \cdot \mathrm{L}^{-1} \cdot \mathrm{s}\end{array}$ & $\begin{array}{c}\text { Mean measured } \\
\text { resistance } \\
\mathrm{kPa} \cdot \mathrm{L}^{-1} \cdot \mathrm{s}\end{array}$ & $\%$ error \\
\hline$R 1$ & 0.80 & 0.87 & +8.7 \\
$R 2$ & 0.96 & 0.98 & +2.1 \\
$R 4$ & 2.22 & 2.25 & +1.4 \\
$R 5$ & 3.28 & 3.22 & -1.8 \\
\hline
\end{tabular}

$R 1-R 5$ : Resistances $1-5$. $R 1$ shows the biggest error, which is probably due to very low pressure changes during ventilation.

element of $300 \mathrm{~mL}$ was inserted between resistor and pump. A series of tests revealed that the Jaeger plethysmograph was able to measure resistances $1-3.5$ $\mathrm{kPa} \cdot \mathrm{L}^{-1} \cdot \mathrm{s}$ with an accuracy of $\pm 2.5 \%$.

\section{Measurement of frequency response characteristics}

Rationale. During plethysmographic measurements, looping between $\Delta V$ box and either $\Delta P$ ao or flow can be caused either by lung function abnormalities or by the physical properties of the equipment. It is therefore essential to be able to check that the equipment itself does not introduce phase lag between signals. Considering this, the ERS-ATS standards specify a fidelity of the equipment of $\pm 10 \%$ in magnitude and $\pm 3^{\circ}$ in phase up to $10 \mathrm{~Hz}[1,2]$. While in most cases this can be assessed traditionally using loudspeakers [11-14], under specialized conditions such as in infant-plethysmographs, where signals of very different magnitude ( $V$ box, $P$ ao, flow) are to be compared and signal transducers are built into the equipment, an infant lung model is advantageous.

Methods. For FRC measurements (Pao versus Vbox) (fig. 5) the infant lung model pump was placed in the plethysmograph and used to ventilate a volume element (e.g. $V 150)$ that was connected to the $P$ ao port. $V 150$ acted as a capacitance, thereby reducing the amplitude of $\Delta P$ ao during ventilatory "efforts" by the piston against the occluded volume outlet so that an appropriate signal to noise ratio could be achieved for both signals.

In order to calculate the effective phase relationship between $V$ box and $P$ ao (i.e. corrected for the frequency response of the model), the model was placed within the box. The pump was then used to generate a pseudorandom noise signal $V \mathrm{p}(\omega)$ ranging $0.25-12 \mathrm{~Hz}$ and the resulting oscillatory pressure changes in $P$ ao and $V$ box were measured $(P$ ao $(\omega)$ and $V$ box $(\omega)$, respectively). The signals were sampled with $1000 \mathrm{~Hz}$ and 12 bit accuracy using the in-built Jaeger AD-board and JScope software. The magnitude and phase relationship were measured from the complex ratio $V \operatorname{box}(\omega) / P$ ao $(\omega)$ and corrected for the influence of the model frequency response function. The frequency response function of the closed model $((V \mathrm{p}(\omega) / P \mathrm{ao}(\omega))$ has been described previously [16]. In this set-up, the model was in series with the plethysmographic equipment. 
a)

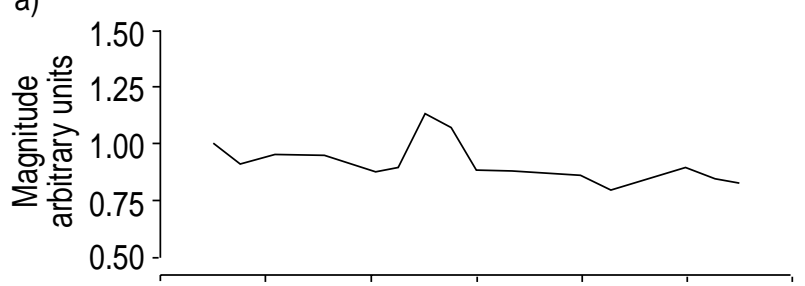

b)

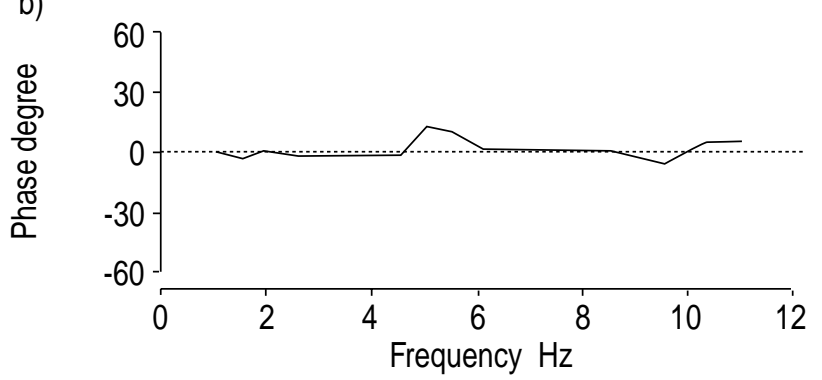

Fig. 5. - Frequency response of functional residual capacity measurements. The relationship box volume $\left(V_{\text {box }}(\omega)\right)$ and airway opening pressure $(P$ ao $(\omega))$ in a plethysmograph after taking the influence of the model frequency response into account mathematically. The magnitude of attenuation (normalized for $V$ box $(\omega))$ in a) arbitrary units; and b) phase shift are plotted against the frequency range $1-11 \mathrm{~Hz}$.

As an analogy for Raw measurements, the model was then placed in the plethysmograph (set-up figure $1 \mathrm{~b}$ ), the pump connected to a volume $(V 300)$ and a resistor element (R5) placed in series with the PNT. V300 acted as a capacitance in series with $R 5$ in order to create measurable changes in $V$ box during these experiments. The effective phase relationship between $V$ box and flow within the plethysmograph was calculated by generating pseudorandom noise, measuring the new magnitude and phase relationship from the complex ratio $V \operatorname{box}(\omega) /$ $V^{\prime}(\omega)$ and then correcting these for the frequency response of the model [16]. During this set-up the model was again placed in series with the plethysmographic equipment.

Results. The results of the frequency response assessments for measurements of FRC and resistance are shown in figure 5 and 6 , respectively. From the corrected signal phase-relationships, it can be seen that the maximum attenuation and phase shifts between $V$ box and either $P$ ao or $V^{\prime}$ were close to the ERS-ATS specifications. The frequency dependent decrease of resistance in figure 6 was not due to any deficiency with respect to frequency response but reflected both the decrease of flow at higher frequencies and the nonlinear pressure/flow relationship of the resistor [16]. Irregularities in both magnitude and phase spectrum (e.g. at $5 \mathrm{~Hz}$ ) were due to signal to noise problems during the measurements in this particular plethysmograph.

In summary. The overall frequency response of an infant plethysmograph can be assessed at up to $10 \mathrm{~Hz}$ using a lung model, even if all signal transducers are in situ and have different pressure ranges. Careful selection of the capacitance and resistance of the
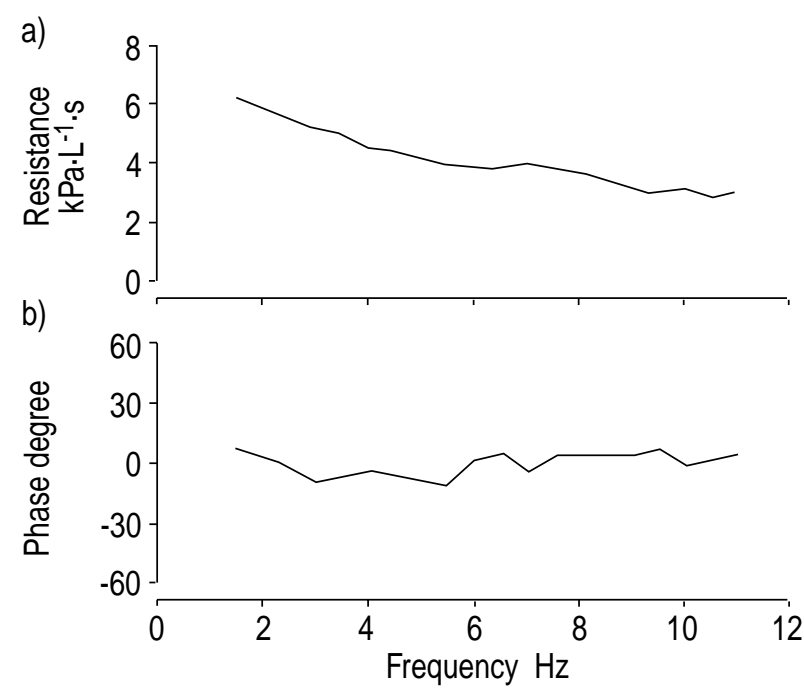

Fig. 6. - Frequency response of resistance measurements. a) magnitude $\left(\mathrm{kPa} \cdot \mathrm{L}^{-1} \cdot \mathrm{s}\right)$ and b) phase relationship of box volume $(V \operatorname{box}(\omega)) /$ flow $\left.V^{\prime}(\omega)\right)$ after taking the frequency response of the model into account mathematically. At low frequencies the magnitude reflects a resistance of $6 \mathrm{kPa} \cdot \mathrm{L}^{-1} \cdot \mathrm{s}$ as was used in this set-up (fig. 1b). At higher frequencies, flow through the pneumotachograph, and hence resistance, decreases. Over the frequency range $1.5-11 \mathrm{~Hz}$, no systemic phase shift appears.

model can be used to adjust for the latter and ensure better signal to noise ratios. However, since the infant lung model will itself introduce some phase shift [16], the frequency response characteristics of the model must be taken into account mathematically when assessing infant lung function equipment.

\section{Discussion}

The results from this study demonstrate that it is possible to use the infant lung model to assess the overall function of an whole-body infant-plethysmograph within the accuracy defined by the ERS-ATS standards $[1,2]$. This includes lung volume measurements, airway resistance measurements and overall frequency response of the plethysmograph. However, the precision of the test device is in turn influenced by various factors such as nonlinear flows due to turbulence in the model, polytropic thermal effects, leak and the mechanical behaviour of moving parts (e.g. piston) of the model. The validity, confounding factors and potential pitfalls of such equipment assessments are discussed below.

\section{Infant lung model volume measurements}

The infant lung model is capable of reproducing model volumes $50-300 \mathrm{~mL}$ which equate to the range of FRC normally encountered in infants up to $10 \mathrm{~kg}$ $[2,8]$. The Jaeger infant-plethysmograph was found to be capable of measuring model volumes accurately from 75-300 mL. However, V50 could not be measured within the limits given by the ERS-ATS standards due to the very small changes in box pressure $(<0.001 \mathrm{kPa})$ 
under these circumstances (fig. 2). The data also demonstrated that such accuracy was only possible if the plethysmograph has a sufficiently long leak time constant (half-life $>10 \mathrm{~s}$ for this particular plethysmograph).

It should be noted that the infant lung model can only assess accuracy under in vitro conditions (i.e. mechanical characteristics of the box, accuracy of software algorithms, etc.). During in vivo measurements, temperature and humidity changes in a plethysmograph due to the presence of an infant may result in drifts of box signal that may adversely affect the accuracy of volume measurements. Such errors, together with any resulting from algorithms designed to correct for thermal drifts, will not be picked up by the model unless an additional source of warmth and humidification is added to the plethysmograph. If other plethysmographs are to be tested, the use of similar adapters with respect to material and volume is recommended in order to take thermal effects into account.

\section{Resistance measurements}

The infant lung model is capable of reproducing resistances $1-5.6 \mathrm{kPa} \cdot \mathrm{L}^{-1} \cdot \mathrm{s}$ which equate to the range of airway resistances normally encountered in infants up to $10 \mathrm{~kg}[2,8]$. By using the model set-up as described under methodology, it was possible to assess how accurately the plethysmograph could measure airway resistance. The optimal set-up of the model should include a volume of $300 \mathrm{~mL}(V 300)$ between the pump and resistor to allow significant $\Delta V$ box without causing any phase shift between $\Delta V$ box and flow up to $120 \mathrm{~min}^{-1}$. It is recommended that the model should be run at a pumping frequency $<60 \mathrm{~min}^{-1}$. The data suggest that the Jaeger infant-plethysmograph is capable of measuring model resistors close to the limits required by the ERS-ATS standards. However, it should be noted that the situation of the model is different from the infant. Whereas in the model, compliance is only related to gas compression, in the infant, lung tissue compliance is additionally important. Thus, to achieve a given pressure change at the model opening, which is comparable to the airway opening pressure in infants, the tidal stroke volume of the model has to be smaller than the tidal volume of a breathing infant. Therefore, during in vitro measurements with the model, $\Delta V$ box changes are smaller than during measurements in infants of similar lung volume. Since the signal to noise ratio of plethysmographic measurements in infants should be better, measurements of low resistances might be more accurate in vivo. The same is potentially true for plethysmographic measurements of very small lung volumes (e.g. $50 \mathrm{~mL})$.

The model was able to evaluate the accuracy with which the mechanical components and software could calculate resistances in vitro. This is, however, only the first step. One of the greatest challenges to practitioners of infant plethysmography has always been the achievement of BTPS conditions during Raw measurements $[20,21]$ since infants cannot be instructed to pant $[8,9]$. In adults, electronic compensation of the $V$ box/ flow relationship has been introduced in an attempt to overcome phase shifts due to temperature and humidity changes of respired gases [22], but this approach has yet to be validated in infants.

\section{Measurement of frequency response characteristics}

Temperature and humidity changes, as well as ventilation inhomogeneities in the diseased infant lung, can cause a phase shift between pressure and flow in an whole-body infant-plethysmograph [8, 9]. Similarly the equipment itself could introduce a phase shift by a combination of the compliant and resistive elements of the equipment (e.g. tubes to pressure transducers or compensation chambers). Fitting algorithms of resistance loops are highly sensitive to the presence of any phase shift. It is therefore essential that any errors due to phase shift introduced by the equipment itself can be ruled out. An infant plethysmograph is just one example of where phase shift plays a critical role for lung function measurements. However, phase shift is a problem in all measurement techniques that are based on two corresponding physiological signals. It should be noted that even if the frequency response of individual components of lung function equipment adhere to the standards, the magnitude and phase relationship between two or more corresponding signals may be altered by the way they are incorporated into the equipment. It is therefore mandatory to test the overall integrated frequency response of any lung function device. This can ideally be done using the infant lung model. It has to be noted that any lung model will itself introduce some phase shift [16]. Thus the frequency response characteristics of the model must be taken into account mathematically when assessing infant lung function equipment.

During FRC manoeuvres, the capacitance of the gas within the box, as well as that within the conducting, tubes, are primarily responsible for any equipment, related phase shift. For Raw measurements, the frequency response of the PNT and attainment of BTPS conditions are additional important factors. Environmental noise is also critical for these measurements and can lead to fluctuations in the frequency response spectrum, as will any thermal effects, which are particularly difficult to assess in an in vitro model.

\section{In conclusion}

An infant lung function model which meets the criteria recently recommended by the European Respiratory Society-American Thoracic Society task force for infants has been developed. It has been shown that the signal to noise error due to the mechanical characteristics does not significantly interfere with the plethysmographic measurements, and that this model can therefore be used to test the accuracy of wholebody infant-plethysmographs with respect to volume and resistance measurements and frequency response. Possible model set-ups that allow such evaluations have been proposed. The performance of this model has been illustrated based on measurements in a commercially available plethysmograph. Based on this limited overall 
evaluation, the Jaeger whole-body infant-plethysmograph appears to fulfil the European Respiratory Society-American Thoracic Society criteria regarding the measurement of model volumes of $75-300 \mathrm{~mL}$, resistance measurements of $1-3.5 \mathrm{kPa} \cdot \mathrm{L}^{-1} \cdot \mathrm{s}$, and frequency response up to $10 \mathrm{~Hz}$ under ambient temperature and pressure, saturated (ATPS) conditions. While this report does not include a detailed protocol for the evaluation of an infant-plethysmograph, it highlights some of the difficulties and pitfalls encountered when undertaking such evaluations, which will hopefully serve as a basis for future developments in this field.

\footnotetext{
Acknowledgements. The authors thank Erich Jaeger GmbH, Hoechberg, Germany for their support, and particularly T. Hilgendorf and I. Dundas for assistance during data collection.
}

\section{References}

1. Frey U, Stocks J, Sly P, Bates JHT. Specifications for signal processing and data handling used in infant pulmonary function testing. Eur Respir $J$ 2000; 16: 1016-1022.

2. Frey U, Stocks J, Coates A, Sly P, Bates JHT. Specifications for equipment used for infant pulmonary function testing. Eur Respir $J$ 2000; 16: 731-740.

3. Bates JHT, Schmalisch G, Filbrun D, Stocks J. Tidal breath analysis for infant pulmonary function testing. Eur Respir J 2000; 16: 1180-1192.

4. Stocks J, Godfrey S, Beardsmore C, Bar-Yishay E, Castile R. Plethysmographic measurements of lung volume and airway resistance. Eur Respir $J$ 2001; 17: 302-312.

5. Sly P, Tepper R, Henschen M, Gappa M, Stocks J. Tidal forced expirations. Eur Respir J 2000; 16: 741748.

6. Morris MG, Gustafsson P, Tepper R, Gappa M, Stocks J. The bias flow nitrogen washout technique for measuring functional residual capacity. Eur Respir $J$ 2001; 17: 529-536.

7. Gappa M, Colin AA, Goetz I, Stocks J. Passive respiratory mechanics: the occlusion techniques. Eur Respir J 2001; 17: 141-148.

8. Stocks J, Marchal F, Kraemer R, Gutkowski P, Bar Yishay E, Godfrey S. Plethysmographic assessment of functional residual capacity and airway resistance. In: Stocks J, Sly PD, Tepper RS, Morgan WJ, eds. Infant respiratory function testing. Lyss, New York, Wiley, 1996; pp. 191-241.

9. Stocks J, Godfrey S. Specific airway conductance in relation to postconceptional age during infancy. $J$ Appl Physiol 1977; 43: 144-154.

10. Miller MR, Pincock AC. Linearity and temperature control of the Fleisch pneumotachograph. $J$ Appl Physiol 1986; 60: 710-715.

11. Jackson AC, Vinegar A. A technique for measuring frequency response of pressure, volume and flow transducers. J Appl Physiol 1979; 47: 462-467.

12. Jackson AC. Dynamic response of transducers used in respiratory physiology Part II. In: Otis $\mathrm{AB}$ ed. Techniques in the Life Sciences. Techniques in Respiratory Physiology, Vol P4/II. Gainesville, Elsevier Scientific, 1984; pp. 411-418.

13. Proulx PA, Harf A, Lorino H, Atlan D, Laurent D. Dynamic characteristics of air-filled differential pressure transducers. J Appl Physiol 1979; 46: 608-614.

14. Vallinis P, Retvalvi S, Davies GM, Coates AL. A simplified method for determining the frequency response of pneumotachographs used in infants. Pediatr Pulmonol 1993; 16: 109-115.

15. Sly PD, Lanteri C, Bates JHT. Effect of the thermodynamics of an infant plethysmograph on the measurement of thoracic gas volume. Pediatric Pulmonol 1990; 8: 203-208.

16. Frey U, Reinmann B, Stocks J. The infant lung function model, a mechanical analogue to test infant lung function equipment. Eur Respir J 2001; 17: 755-764.

17. Bargeton D, Barrès G. Time characteristics and frequency response of body plethysmography. In: DuBois $\mathrm{AB}$ and van de Woestijne KP, eds. Progress in Respiration Research. Body Plethysmography. Basel: Karger, 1969, vol. 4, 2-23.

18. Schuessler TF, Bates JHT. A computer-controlled research ventilator for small animals: Design and evaluation. IEEE Trans Biomed Eng 1995; 42: 860-866.

19. Bland JM, Altman DG. Statistics Notes: Measurement error proportional to the mean. BMJ 1996; 313: 106.

20. Peslin R, Duvivier C, Vassiliou M, Gallina C. Thermal artifacts in plethysmographic airways resistance measurements. J Appl Physiol 1995; 79: 1958-1965.

21. Peslin R, Duvivier C, Malvestio P, Benis AR, Polu JM. Frequency dependence of specific airway resistance in a commercialised plethysmograph. Eur Respir J 1996; 9: $1747-1750$.

22. Peslin R, Duvivier C, Malvestio P, Benis AR. Correction of thermal artifacts in plethysmographic airway resistance measurements. J Appl Physiol 1996; 80: 2198-2203. 